\title{
Real-time Health Monitoring System Using Wireless Body Area Network
}

\author{
a, * Fahad Khan, ${ }^{\mathrm{b}}$ Ateeq Ur Rehman, ${ }^{\mathrm{c}}$ Jawad Khan, ${ }^{\mathrm{d}}$ Muhammad Tariq Sadiq, ${ }^{\mathrm{e}}$ Rao Muhammad Asif, \\ a Zahid Mahmood

\begin{abstract}
a: Department of Electrical and Computer Engineering, COMSATS University Islamabad, Abbottabad Campus, Pakistan b: Department of Electrical Engineering, GC University Lahore, Pakistan

b: College of Internet of Things Engineering, Hohai University, Changzhou 213022, China

c: Department of Computer Sciences, COMSATS University Islamabad, Abbottabad Campus, Pakistan

d: School of Automation, Northwestern Polytechnical University, 127 West Youyi Road, Xian, 710072, China

e: Faculty of Electrical Engineering, The Superior College (University Campus), Lahore, Pakistan

*Corresponding Author Email: fahadkhan@ cuiatd.edu.pk
\end{abstract}

\begin{abstract}
Wireless body area network (WBAN) being a sub-domain of wireless sensor network (WSN) is a new emerging technology for healthcare applications. A WBAN consists of low-power tiny wireless nodes placed on or around the human body that continuously observe vital health signs of a patient. These sensors are capable of sending information of physiological parameters taken from human body to other devices for diagnosis procedures and prescription. WBAN provides ubiquitous healthcare services and enables greater mobility without restricting human normal activities, as the medical personnel can observe the patient health conditions based on the data received through the wireless network. This research work provides a WBAN based healthcare monitoring system that can provide the electrocardiogram (ECG), heartbeat, and human body temperature information. The wireless transmission of the received data from human body is performed by using Zigbee IEEE802.15.4 communication standard. The physiological data will be communicated to remote medical server where data is stored and analyzed. In case any disease is diagnosed, medical personnel can provide immediate assistance to the patients.
\end{abstract}

Keywords- WBAN, ECG, Heartbeat, WBAN prototype, WBANs Open issues.

Date Received 21 Mar 2020

Date Accepted 11 May 2020

Date Published 25 June 2020

\section{INTRODUCTION}

$\mathrm{W}$ ireless sensor network (WSN) is widely utilized in numerous fields for monitoring and surveillance purposes including healthcare, environment monitoring, and military training. WSN comprises of sensor nodes deployed to monitor some physical conditions with computational and communication capabilities under scarce energy resources [1][3]. The on-going increase in portable monitoring devices and location independent monitoring facility has resulted in the utilization of WSN for biomedical purposes [4]. This emerging application of WSN for biomedical services is called as wireless body area network (WBAN) [5].

A WBAN consists of in-body and on-body low-power tiny wireless nodes that continuously observe vital health signs of a patient for diagnosis procedures and prescription [6]. WBAN provides ubiquitous healthcare, improved quality of life, and treatment success rates in humans. WBAN enables greater mobility without restricting human normal activities where doctor can observe the patient medical conditions based on the data received through the wireless network.

A typical architecture of WBAN based healthcare system is shown in Fig. 1 [7], which comprises of several actuators or sensor nodes and a body control unit (BCU). The duty of BCU is to gather physiological data from the nodes and transmits this information to the remote medical server. The medical personnel provide necessary medical services after analyzing the received information. Several wireless communication technologies are available for transmission of sensor information to the remote healthcare centre such as $\mathrm{WiFi}$, WiMAX, Bluetooth, Zigbee, existing mobile data networks $(4 \mathrm{G} / 5 \mathrm{G})$, etc. However, license free ISM band $(2.4 \mathrm{GHz}-2.4835$ GHz) [8] like Zigbee and Bluetooth are the dominant standards being deployed to accomplish data communication in WBAN. Zigbee is also a widely used technology in WSN based applications for smart grid systems [9]. 


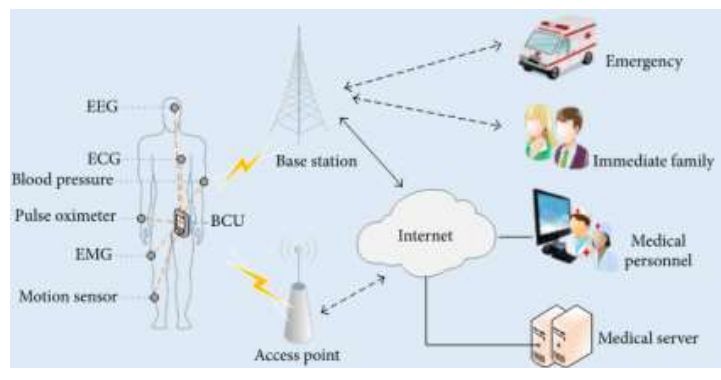

Fig. 1. An overview of WBAN communication architecture.

WBAN is still facing several technological issues for its widespread use in medical applications and researchers have shown several open issues related to WBAN implementation in healthcare applications such as overall system efficiency and data reliability [10], [11].

In this paper, hardware design of WBAN based medical system for monitoring real-time heart conditions and body temperature using Zigbee is presented. The gathered information from the WBAN is forwarded to the remote medical server using GUI where professionals will analyze the data and make appropriate medical decisions. Some open issues in WBANs and their utilization in ECG and medical applications are also highlighted.

The rest of the paper is organized as follows. Section II presents background and related work. The hardware design and results of proposed WBAN monitoring system for health applications is discussed in Section III followed by conclusions in Section IV.

\section{BACKGROUND AND RELATED WORK}

WBAN consists of small size and low-power sensor nodes that have limited computational and storage capabilities. WBAN sensors are usually implanted in or placed on human body to monitor different essential health parameters, such as electroencephalography (EEG), electrocardiogram (ECG), photoplethysmogram (PPG), Motor Imagery EEG [12], pulse rate, enabling most widely used solution in healthcare area [11]. The collected data from deployed sensors or actuators is then transferred to remote medical server using wireless communications technology where diagnostic and therapeutic decisions are performed for a medical treatment.

Undoubtedly, the advancement in wearable sensors along with wireless communication technologies has provided prevention and detection of fatal disorders. In general, wearable sensor in healthcare applications consists of following types:

- $\quad$ Electromagnetic tracking system (ETS) sensor is used as a body position measurement sensor [13].

- Accelerometer sensors or motion detection sensors are used to sense acceleration [14].

- EMG sensor that provides electrical activities of particular muscle.

- ECG sensor allows interpretation of electrical activity of heart.

- EEG sensor is used to measure brain waves of a person.

- Blood glucose monitoring sensor enables to measure blood glucose level (BGL).
- Ground reflection force (GRF) sensor that is used in gait analysis.

Low-power operation and miniaturization are two essential physical requirements of WBAN sensor nodes since these two factors determine the lifetime of the devices and their suitability to be implanted on the human body. In literature, several useful works have been conducted in the development of WBANs. A smart phone based application for the detection of heart attack is presented in [15], where index fingers are placed on mobile camera to calculate the heart rate. In [16], non-contact capacitive electrodes are used to monitor heart activity through clothes, for which results reveals heart error rate within $1 \%$.

An excellent survey related to wearable sensors in WBANs is provided in [17]. The authors have discussed medical applications (ECG, EEG, detection of blood glucose level, respiration rate, etc) and non-medical application (daily exercise monitoring) of several types of wearable sensors. They have also provided a detailed analysis of filtering techniques for different types of noise present in ECG signals. To extract ECG features from WBAN sensors, the first step is to remove noise content from ECG signal using pre-processing technique. The noise component present in ECG signal are predominant in the range of 0.01-150 Hz. Table I summarizes important type of noise present in ECG signal.

Microwave and radio communications enable high bandwidth and large range. However, in near-body health applications, microwave and radio communications should be avoided due to the high exposure of radiations to body tissues [18]. Moreover, electromagnetic interferences (EMI) of RF devices cause serious malfunctions of medical equipment [19]. In such cases, ultrasonic communication is a more suitable due to its less radiation effect on human body. In [20], Galvanic Skin Response (GSR) sensor is used to detect whether a person is under stress or not. The GSR data is transmitted using ultrasonic communication system with an amplitude shift keying (ASK) modulation. The results reveal that the proposed ultrasonic based GSR sensor performs well in relatively short range and narrow beamwidth. Wireless optical communication (WOC) based WBAN systems have also emerged an alternative way to reduce electromagnetic pollution in hospital environments. Generally, WBANs based health monitoring applications need low data rates [21]. A WBAN system based on wireless optical communication (WOC) is investigated in [22], which provides a health monitoring setup composed of three nodes and a coordinator, and an optical code division multiple access (OCDMA) scheme is utilized. In [22], authors have evaluated the performance of WOC based WBAN system for medical requirements such as data rate, power consumption and flexibility, and it is shown that WOC has the ability to provide a alternative to RF based WBAN systems particularly in scenarios where there are RF interference concerns.

TABLE I

Different types of noise in ECG signal

\begin{tabular}{|l|l|}
\hline Noise & Frequency \\
\hline Power line interference & $50 \mathrm{~Hz}$ or $60 \mathrm{~Hz}$ \\
\hline Baseline wandering & Below $0.5 \mathrm{~Hz}$ \\
\hline
\end{tabular}




\section{DOI: https://doi.org/10.36785/BUITEMS.JAES.332}

\begin{tabular}{|l|l|}
\hline Electrodes motion artifacts & $1 \mathrm{~Hz}$ to $10 \mathrm{~Hz}$ \\
\hline Electromyographic (EMG) noise & $25 \mathrm{~Hz}$ to $100 \mathrm{~Hz}$ \\
\hline
\end{tabular}

In [23], a continuous vital signs monitoring system (VSMS) using WSN where node architecture is based on multiple routers that are used to provide path for sensor nodes to transmit data or receive data from the observation room [24].

in hospital is proposed. Blood pressure, heartbeat, and temperature are monitored and forwarded to observation room

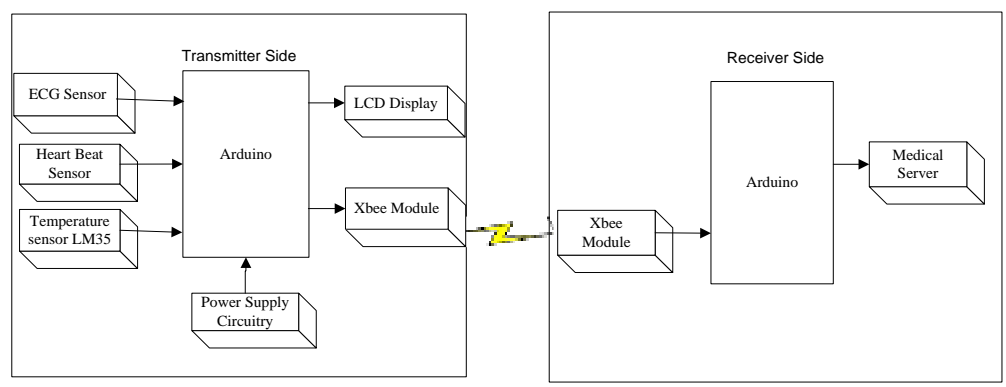

Fig. 2. Block diagram of WBAN based healthcare system.
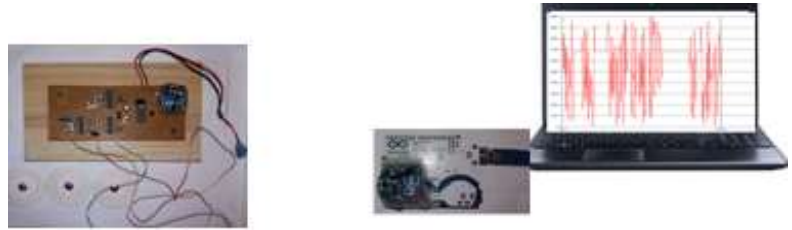

Fig. 3. Hardware setup of WBAN based healthcare system for ECG, heartbeat, and temperature monitoring.

Energy efficiency, which is an important requirement of sensing nodes, is achieved by selection of the optimal routes in WSN through genetic algorithm (GA) [23]. A detailed discussion on reliable communication and security challenges in WBANs is provided in [25]-[27].

The next section provides the design of WBAN for real-time monitoring of ECG, body temperature, and heartbeat.

\section{DESIGN OF WBAN FOR HEALTH MONITORING APPLICATIONS}

\section{A. Block Diagram of Proposed WBAN System}

ECG sensor (LM324), heartbeat sensor (LM358), and body temperature sensor are used to measure vital parameters that are monitored from a human by attaching electrodes on human body. Fig. 2 shows the block diagram of WBAN based ECG, heartbeat, and human-body temperature monitoring system.

The ECG, heartbeat, and body temperature signals have very low amplitude usually in $0.5-1 \mu \mathrm{V}$, which by setting desired gain is amplified to the value of 4.5-5 mV. Another problem in ECG analysis is the noise mixed with ECG signals, for removal of noise the ECG and body temperature signals are passed through a bandpass filter, hence to allow a specific band of frequencies while eliminating unwanted frequencies, also a notch filter is used to remove power line interference at $50 \mathrm{~Hz}$. The physiological signals i.e. ECG and temperature are analog signals, which are then digitized using A/D convertor. The digitized signal is then processed and stored in Arduino [28]. The Arduino will then forward the stored ECG, heartbeat, and body temperature via Zigbee to the remote medical server for data record keeping and medical service in case any abnormality is diagnosed.

\section{B. Hardware and Result Analysis for Proposed WBAN System}

The hardware implementation of proposed WBAN based healthcare system for ECG, heartbeat rate, and temperature monitoring is provided in Fig. 3. This sensing module is attached to human body and the body essential health data will be communicated to remote medical server.

By attaching sensing module to human body, the real-time ECG, heartbeat, and temperature are obtained for a patient with ID\#122APAK using GUI at remote laptop as shown in Fig. 4. The result obtained in Fig. 4 provides the following useful information about the patient:

- A normal ECG is shown in Fig. 4, which reveals that the heart beats rhythmically at 60-100 beats per minute. The readings strongly resemble a normal ECG signal. The R, S, and T segments of ECG are visible and resemble a normal ECG. In a normal ECG case, the PQRST curve repeats after every 0.87 interval. However, in abnormal faced by heart, this time may increase or decrease.

- In normal scenarios, heartbeat rate for adults ranges from $60-100$ beats a minute. Heartbeat rate is also depicted in Fig. 4. In case heartbeat does not show normal values, hospital staff can provide in time medication to avoid heart disorders.

- The value of body temperature depicted in Fig. 4 is 38 Celsius, which is a normal body temperature. 


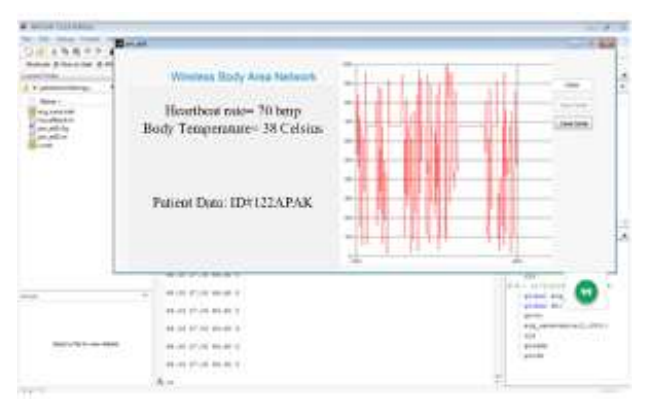

Fig. 4. GUI for for ECG, heartbeat rate, and body temperature obtained at remote Laptop.

\section{Challenges in WBAN for Healthcare Applications}

The healthcare related applications require a proper design of WBANs. The senor nodes and coordinator in WBAN are limited in terms of energy. For critical health data, lifetime of nodes is crucial to provide real-time health data. The proper utilization of technique such as multiple transmission scheduling and efficient channel access schemes at the MAC layer can prolong the battery of nodes in WBAN. The WBANs reliability is also an important concern, which can be increased by reducing transmission delays and packet loss in the network through effective utilization of appropriate packet size and channel access schemes at MAC layer.

Other than these challenges, the layer specific issues are also a major concern in WBAN applications. For example, quality of service for emergency traffic, network scalability, and energy-efficient routing protocols needs to be incorporated in WBAN medical applications for its efficient functionality. The noise from different sources such as electrodes and wirings connected to WBAN degrades performance. The data security, privacy, and interoperability need a considerable attention for proper operation of WBAN healthcare system. Some of the challenges and open research issue related to WBANs for healthcare applications are depicted in Fig. 5.

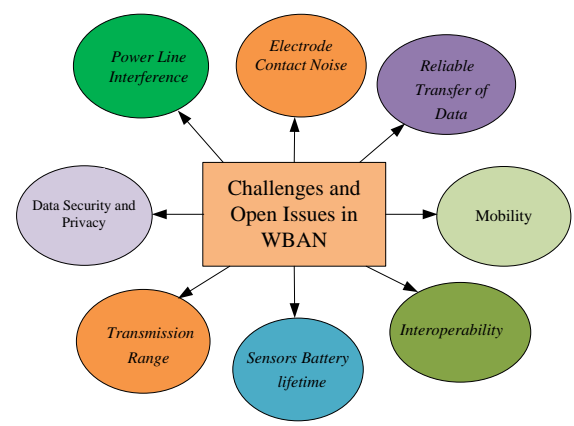

Fig. 5. Challenges and open research issues in WBAN.

\section{CONCLUSIONS}

Smart healthcare devices have motivated researchers to provide solutions to improve health and social care provisions to existing medical services. In this perspective, WBAN is a potential technology that can revolutionize the medical and healthcare sectors. In this paper, WBAN based healthcare system is developed to obtain vital signs from human body that can provide benefits to both patients and doctors. Moreover, the developed WBAN system can provide quick and fast response for emergency situations, such as, abnormal ECG, heartbeat, etc. In future, the authors are interested to design a monitoring healthcare portable device for athletes and sportsmen.

\section{REFERENCES}

[1] J. D. Culler, D. Estrin, M. Srivastava, "Overview of Sensor Networks," IEEE Computer, USA, vol 37, pp. 41-49, August 2004.

[2] F. Khan, F. Shahid, S. B. Hassan and A. Rashid, "Stable Topology Based Energy Efficient Protocol for Distributed Network for Heterogeneous Wireless Sensor Applications," 2015 13th International Conference on Frontiers of Information Technology (FIT), Islamabad, 2015, pp. 144149.

[3] A. Yousaf, F. Ahmad, S. Hamid and F. Khan, "Performance Comparison of Various LEACH Protocols in Wireless Sensor Networks," 2019 IEEE 15th International Colloquium on Signal Processing \& Its Applications (CSPA), Penang, Malaysia, 2019, pp. 108-113.

[4] Chris Otto, Aleksandar Milenković, Corey Sanders, Emil Jovanov, "System Architecture of a Wireless Body Area Sensor Network for Ubiquitous health monitoring," Journal of Mobile Multimedia, Vol. 1, No.4, 2006, pp. 307-326.

[5] Bahae Abidi, Abdelillah Jilbab \& El Haziti Mohamed, "Wireless body Area Network for Health Monitoring," Journal of Medical Engineering \& Technology, 43:2, pp. 124-132, 2019.

[6] Ullah S, Higgins H, Braem B, et al. A Comprehensive Survey of Wireless Body Area Networks," J Med Syst. 2012;36:1065-1094.

[7] M. Chen, S. Gonzalez, A. Vasilakos, H. Cao, and V. C. M. Leung, "Body Area Networks: A Survey," Mobile Networks and Applications, vol. 16, no. 2, pp. 171-193, 2011.

[8] A. U. Rehman, M. T. Sadiq, N. Shabbir, and G. A. Jafri, "Opportunistic Cognitive MAC (OC-MAC) Protocol for Dynamic Spectrum Access in WLAN Environment," Int. J. Comput. Sci. Issues, vol. 10, no. 6, pp. 45$51,2013$.

[9] H. Ali, W. Y. Chew, F. Khan and S. R. Weller, "Design and Implementation of an IoT Assisted Real-Time Zigbee Mesh WSN Based AMR System for Deployment in Smart Cities," 2017 IEEE International Conference on Smart Energy Grid Engineering (SEGE), Oshawa, ON, 2017, pp. 264-270.

[10] Mark AH, Harry CP, Jr, Adam TB, Kyle R, Benton HC, James HA, John L. Body Area Sensor Networks: Challenges and Opportunities," IEEE Computer Society; Atlantic City, NJ, USA: 2009. pp. 58-65.

[11] V. Custodio, F. J. Herrera, G. López, and J. I. Moreno, "A Review on Architectures and Communications Technologies for Wearable HealthMonitoring Systems," Sensors, vol. 12, no. 10, pp. 13907-13946, 2012.

[12] M. T. Sadiq et al., "Motor Imagery EEG Signals Classification Based on Mode Amplitude and Frequency Components Using Empirical Wavelet Transform," in IEEE Access, vol. 7, pp. 127678-127692, 2019.

[13] F. Raab, E. Blood, T. Steiner, and H. Jones, "Magnetic Position and Orientation Tracking System," Aerospace and Electronic Systems, IEEE Transactions on, no. 5, pp. 709-718, 1979.

[14] A. Godfrey, R. Conway, D. Meagher, and G. O' Laighin, "Direct Measurement of Human Movement by Accelerometry," Medical engineering \& physics, vol. 30, no. 10, pp. 1364-1386, 2008.

[15] M. Ashrafuzzaman, M. M. Huq, C. Chakraborty, M. R. M. Khan, T. Tabassum, and R. Hasan, "Heart Attack Detection using Smart Phone," International Journal of Technology Enhancements and Emerging Engineering Research, vol. 1, pp. 23-27, 2013.

[16] Y. M. Chi and G. Cauwenberghs, "Wireless Non-contact EEG/ECG Electrodes for Body Sensor Networks," 2010 International Conference on Body Sensor Networks, Singapore, 2010, pp. 297-301.

[17] A. Rahim, N. Javaid, M. Aslam, Z. Rahman, U. Qasim and Z. A. Khan, "A Comprehensive Survey of MAC Protocols for Wireless Body Area Networks," Broadband, Wireless Computing, Communication and Applications (BWCCA), 2012 Seventh International Conference on, Victoria, BC, 2012, pp. 434-439.

[18] E. J. Escudero and G. S. Rai, "An Investigation into Ultrasonic Communication for Near-Body Networks," 2011.

[19] Periyasam, M.; Dhanasekaran, R., "Electromagnetic interference on critical medical equipments by RF devices," Communications and Signal Processing (ICCSP), 2013 International Conference on , vol., no., pp.78,82, 3-5 April 2013. 


\section{DOI: https://doi.org/10.36785/BUITEMS.JAES.332}

[20] M. H. B. Pratama, A. Munandar, K. Mujib, E. S. Wicaksono and A. A. Zahra, "Implementation of Ultrasonic Communication for Wireless Body Area Network using Amplitude Shift Keying Modulation," 2016 IEEE Region 10 Conference (TENCON), Singapore, 2016, pp. 3790-3793.

[21] M. Paksuniemi, H. Sorvoja, E. Alasaarela, and R. Myllyla, "Wireless Sensor and Data Transmission Needs and Technologies for Patient Monitoring in the Operating Room and Intensive Care Unit," 27th Annual International Conference of the Engineering in Medicine and Biology Society, 2005, pp. 5182-5185.

[22] L. Chevalier, S. Sahuguède and A. Julien-Vergonjanne, "Wireless Optical Technology based Body Area Network for Health Monitoring Application," 2015 IEEE International Conference on Communications (ICC), London, 2015, pp. 2863-2868.

[23] S. W. A. Hashmi, B. A. Alvi, M. Rehan, M. S. Kamal, and M. Y. Zaheen, "Energy Efficient Vital Signs Monitoring System (VSMS) using Wireless Sensor Networks," Wireless Personal Communications, vol. 76, no. 3, pp. 489-501, Mar 2014.

[24] Arslan Haider, Ateeq Ur Rehman, Noman Shabbir, Syed Rizwan Hassan, and Irfan Haider, "A Three Stage Load Sharing Routing Algorithm to Increase Lifetime of Cognitive Radio Sensor Networks", in Journal of Communications, Vol. 12, No. 5, ISSN: 1796-2021, May 2017.

[25] R. A. khan, and A.-S. K. Pathan, "The State-of-the-Art Wireless Body Area Sensor Networks: A Survey," International Journal of Distributed Sensor Networks, vol. 14, no. 2, 2018.

[26] M. S. Taha, M. S. M. Rahim, M. M. Hashim, F. A. Johi, "Wireless Body Area Network Revisited, ," Int. J. Eng. Technol., vol. 7, no. 4, pp. 34943504, 2018.

[27] G. Mehmood, M. Z. Khan, S. Abbas, M. Faisal and H. U. Rahman, "An Energy-Efficient and Cooperative Fault- Tolerant Communication Approach for Wireless Body Area Network," in IEEE Access, vol. 8, pp. 69134-69147, 2020.

[28] A. M. Furqan Durrani, A. U. Rehman, A. Farooq, J. A. Meo and M. T. Sadiq, "An Automated Waste Control Management System (AWCMS) by Using Arduino," 2019 International Conference on Engineering and Emerging Technologies (ICEET), Lahore, Pakistan, 2019, pp. 1-6. doi: 10.1109/CEET1.2019.8711844.

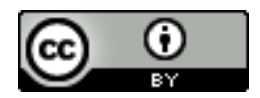

Journal of Applied and Emerging
Sciences by BUITEMS is licensed under a Creative
Commons Attribution 4.0 International License.

